\title{
A laboratory-based study on patients with Parkinson's disease and seborrheic dermatitis: the presence and density of Malassezia yeasts, their different species and enzymes production
}

Valentina S Arsic Arsenijevic ${ }^{*}$, Danica Milobratovic ${ }^{2}$, Aleksandra M Barac ${ }^{1}$, Berislav Vekic ${ }^{3}$, Jelena Marinkovic ${ }^{4}$ and Vladimir S Kostic ${ }^{5}$

\begin{abstract}
Background: Seborrheic dermatitis (SD) and Parkinson's disease (PD) are frequently associated conditions. Aims of this study were: to determine severity of SD, presence of different species and density of Malassezia yeasts; to assess yeast lipases and phosphatases production in vitro and to compare these results between SD patients with and without PD.

Methods: This case-control prospective study was conducted at the Dermatology and Neurology Units, Clinical Centre of Serbia and at the National Medical Mycology Reference Laboratory, University of Belgrade Medical School, Serbia. A total of 90 patients and 70 healthy controls (HC) were investigated: 60 patients with SD (SDN) and 30 patients with SD and PD (SDP). Culture-based mycological examination was carried out on lesional skin (LS) and non-lesional skin (NLS). A yeasts density was determined by counting the Malassezia colony forming units per tape (CFU/tape). Enzymes production by isolated Malassezia was investigated.

Results: The most patients with SD were male (76.7\%; SDP and 63.3\%; SDN) and the intensity of SD was dominantly severe or moderate (76.7\%; SDP and 75\%; SDN). The presence of Malassezia was high on LS in both groups (87.3\%; SDP and $86.7 \%$; SDN) ( $p=0.667)$.

The highest yeasts density (mean CFU/tape $=67.8$ ) was detected on LS in 53\% of SDP group and in $21.7 \%$ of SDN group (mean CFU/tape=31.9) $(p<0.01)$. The presence of negative cultures was lower in SDP group (13.3\%) in comparison to HC and SDN groups (37\% and 31.7\%, respectively). Malassezia density on NLS in SDP group (mean CFU/tape=44.3) was significantly higher in comparison to SDN and HC $(p=0.018)$. M. globosa was the most abundant species identified amongst isolates from the SDP group (42.3\%) and exhibited high production of phosphatase and lipase in vitro.

Conclusion: From this laboratory-based study a positive correlation between SD, PD, M. globosa incidence, high yeast density and high phosphatase and lipase activity was established. Our data lead to conclusion that local skin performance of PD patient's characterized with increased sebum excretion ratio play a role in SD by stimulation of yeasts replication and enzyme production.
\end{abstract}

Keywords: Seborrheic dermatitis, Parkinson's disease, Malassezia species, Colony forming units, Enzymes

\footnotetext{
*Correspondence: mikomedlab@yahoo.com

${ }^{1}$ Institute of Microbiology and Immunology, University of Belgrade Medical

School, Dr Subotica 1, Belgrade, Serbia

Full list of author information is available at the end of the article
} 


\section{Background}

Parkinson's disease (PD) is the second most common neurodegenerative disorder which affects approximately seven million people globally and one million people in the United States [1]. The prevalence of PD is about $0.3 \%$ of the whole population in industrialized countries, $1 \%$ in the elderly over 60 years of age and $4 \%$ of the population over 80 years [2]. "Young-onset PD" appears between ages 21 and 40 years with greater probability for developing various adverse effects of treatment, and deterioration of the quality of life [3].

Patients with PD, in addition to motor system disorders, often suffer from non-motile symptoms, which can include decreased facial skin motility resulting in a characteristic hypomimic facial expression, which has an impact on their quality of life [4]. This condition is associated with seborrheic dermatitis (SD) and increased sebum excretion ratio (SER) in almost $60 \%$ of the cases most likely due to the parasympathetic system hyperactivity as well as to the systemic effect of Melanocyte-stimulating hormone (MSH) $[4,5]$.

SD is chronic skin disease presented with typical sharply demarcated red patches and plaques with greasy scales in areas with increased density of sebaceous glands, namely the scalp, face, hairline, eyebrow, glabella, nasolabial folds, ears, upper trunk and flexures. SD affects approximately $3-5 \%$ of the population [6]. The intensity of symptoms, including inflammation and itching, varies with more severe clinical presentation occurring in predisposed groups. SD prevalence was found to be higher in some medical conditions including HIV positive and AIDS patients (34-83\%) [7] and PD patients (52-59\%) [8]. Endogenous and exogenous conditions, such as hormones (androgens), increased SER, altered immune response and neurological factors $[9,10]$ were found to play an important role in the pathogenesis of SD, but the exact mechanisms are still to be elucidated.

Evident is recent increased research interest in lipophilic Malassezia yeasts. Genus Malassezia now contains 14 species, among which 7 are well characterized culturally and biochemically [11]. Members of this genus are part of normal human flora, however they are also thought to be exacerbating factor in a number of skin conditions, such as pityriasis versicolor, Malassezia folliculitis, SD, atopic dermatitis and even some life threatening nosocomial bloodstream infections [12,10]. Malassezia species and their skin density vary in different populations [13], countries, age groups and genders, as well as between normal, non-lesional skin (NLS) and lesional skin (LS) $[14,11]$. For currently unknown reason, these yeasts can change their saprophytic state and invade stratum corneum of the skin as pathogens.

Although SD is a multifactorial disease, there are two major factors in developing SD: the increased production of host sebum and the presence and increased reproduction of Malassezia yeasts. The presence and density of Malassezia yeasts, different species and production of enzymes in PD patients have not been investigated yet, thus the aim of this study was to determine the following: (I) the severity of SD in study group, (II) the presence and density of Malassezia and (III) enzyme production in isolated species in patients with SD and PD.

\section{Methods}

\section{Source population}

PD and SD patients treated at the Neurology and the Dermatology Units, Clinical Centre of Serbia from January to June 2011 were included in this study.

\section{Study population}

There were 90 patients with SD, 21 to 82 years old, (61 men, 29 women; mean age $54 \pm 17.5$ years), among those 30 patients suffered from PD and SD at the same time (SDP group, 23 men, 7 women; mean age $67 \pm 7$ years) while 60 patients had SD only (SDN group, 38 men, 22 women; mean age $48 \pm 18$ years). Healthy control (HC) group were persons without $\mathrm{SD}(\mathrm{n}=60)$ randomly selected and matched by age and sex (44 men, 26 women; mean age $49 \pm 19.5$ years). HC had apparently normal facial skin, without any evidence of dermatitis. None of these patients had received topical or systemic antifungal therapy or corticosteroids for at least 4 weeks prior to sampling. All patients signed informed consents.

\section{Study design}

This was a prospective case-control study which was conducted at the National Reference Medical Mycology Laboratory, Faculty of Medicine, University of Belgrade (laboratory-based investigation) and at Neurology and the Dermatology Units, Clinical Center of Serbia (clinicallybased investigation). Determination of SD severity and laboratory-based investigation was done in order to determine the Malassezia yeast presence and their density, type and enzymes production.

\section{Determination of SD severity}

A clinical assessment of SD was carried out and scored. The overall SD score was calculated by scoring the severity of SD (from 1 to 12 ) and the facial skin area affected (from 1 to 5). The severity of SD was evaluated according to the presence of: erythema, scale, infiltration and pustule. A four-point score was used for each parameter (0-absent, 1-mild, 2-moderate, 3-severe). The second scale was based on the percentage of the affected skin area: less than $10 \%$ (1 point), $10-30 \%$ ( 2 points), $30-$ $50 \%$ (3 points), $50-70 \%$ (4 points) and over $70 \%$ (5 points). The result was obtained by multiplying the scores of both scales giving an overall score: mild SD (5 or less); 
moderate SD (6 to 11) and severe SD (from 12 to 60). Severity of SD has been described as: mild (the total score $\leq 5$ ); moderate (the total score between 6 and 11); and severe (the total score between 12 and 60) [15].

\section{Sampling procedure, isolation and quantification of Malassezia yeast}

Three skin samples were taken from SD patients' foreheads, from LS and NLS, as well as from normal-looking skin from the foreheads of $\mathrm{HC}$. The sampling was done by stripping $4 \mathrm{~cm}^{2}$ tape (Superabsorb ${ }^{\oplus}$ F, Lohmann Rauscher) [16]. Each tape was transferred to Dixon and LeemingNotman agar (LNA) in order to isolate Malassezia lipophilic yeast and to Sabouraud agar in order to isolate non-lipophilic yeasts $M$. pachydermatis or Candida. Plates were incubated at $32^{\circ} \mathrm{C}$ for 10 days and observed daily. The cultures were considered positive for Malassezia if there was growth on Dixon and LNA. Yeasts isolates were quantified and identified using morphological and biochemical tests. The number of Malassezia colonies was counted and the growth densities were expressed as the mean number of colony forming units per tape (mean CFU/tape). The Malassezia density (CFU/tape) was performed of Dixon and LNA media and ranked into three levels: low (CFU/tape $1 \leq 19)$, medium (CFU/ tape $=20-40)$ and high (CFU/tape > 40) [13]. Production of enzymes from the protease, lipase, phosphatase and glycosidase classes was determined for selected isolates.

\section{Identification of Malassezia yeasts}

Seven lipophylic Malassezia species were identified: M. globosa, M. furfur, M. slooffiae M. sympodialis, $M$. restricta, $M$. obtusa and $M$. japonica based on microscopic characteristics, presence of catalase and the ability of each isolate to utilize different Tweens [17]. The presence of catalase was determined by applying a drop of $10 \%$ hydrogen peroxide on a culture smeared on a glass slide. The production of gas bubbles indicated a positive reaction. The ability to utilize different Tween substrate was assessed on the agar plates, by spreading fresh yeast suspension onto plates. Tween 20, 40, 60 and $80(5 \mu \mathrm{l})$ were added in 4 wells ( $2 \mathrm{~mm}$ diameter) in each plate. The plates were incubated at $32^{\circ} \mathrm{C}$ for one week and the growth of yeast around individual wells contain different Tween was recorded and Malassezia were identified (Figure 1).

\section{Determination of Malassezia yeasts enzyme production by ApiZym assay}

The activity of 19 different enzymes produced by Malassezia was tested using the API Zym assay (bioMerieux, Marcy l'Etoile, France). A total of 26 Malassezia isolates from SD groups were tested for: proteases $(n=5)$, lipases $(n=3)$, phosphatases $(n=3)$, glycosidases $(n=8)$. Results were expressed semi-quantitatively, ranging from no activity to maximum enzymatic activity.

\section{Data collection}

All subjects had a complete history, physical examinations and necessary procedures from skin were done to confirm the diagnosis. Trained mycologists carried out laboratory analysis and were engaged in data collection and data entry.

\section{Data analysis}

Statistical analysis was done using the Statistical Package for Social Science (SPSS) program version 15.0. T-test was used to analyze the differences between severity of disease and demographic data; Mann-Whitney and Kruskal Wallis tests were used to analyze the median number of Malassezia CFU/tape within the same groups on LS and NLS and the median number of Malassezia CFU/tape from all patients with SD and HC. Chi-square tests were performed to determine the differences in: (I) the prevalence of different species of Malassezia in SDN and SDP groups, (II) the prevalence of different species of Malassezia in all patients with SD and HC. The relationship between study groups and culture-based mycological findings was determined by logistic regression analyses adjusted on sex and age.

The study was approved by the Ethical Board of the School of Medicine, University of Belgrade, and the Clinical Center of Serbia (No 5030/5). The anonymity of individuals was preserved during the study.

\section{Results}

Patient's demographic data and determination of SD severity

Most SD patients were male (77\%; SDP and 63\%; SDN) $(\mathrm{p}<0.01)$ and intensity of SD was dominantly in severe or moderate form (76.7\%; SDP and 75\%; SDN). Taking into account SD categories, there was no statistically significant difference between SDP and SDN groups in terms of SD severity: mild (23.3\%; SDP and 25\%; SDN); moderate (60\%; SDP and 45\%; SDN); severe (17\%; SDP and $30 \%$; SDN) ( $\mathrm{p}=0.175$ ) (Table 1). However, when SD was measured by scale there was a significant difference between SDP and SDN patients (OR 0.81; 95\%CI = 0.660.99; $\mathrm{p}=0.043)$.

The correlation between presence and growth density of Malassezia yeasts on LS and NLS

Comparison of Malassezia counts (CFU/tape) on LS and NLS between studied groups was carried out. The correlation between negative cultures and growth density of Malassezia yeasts on LS and NLS is shown on Table 2. The presence of Malassezia was high in LS in both groups (87.3\%; SDP and 86.7\%; SDN) ( $\mathrm{p}=0.667)$. The highest yeasts density (mean CFU/tape $=67.8$ ) was detected on 


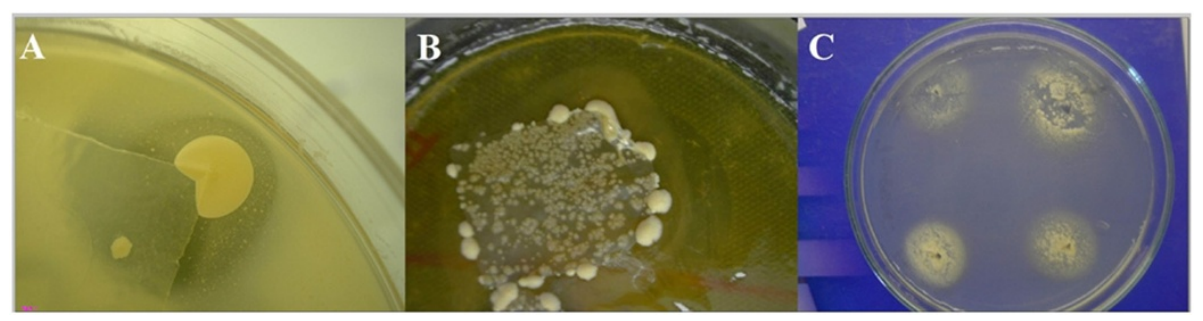

Figure 1 Pictures of isolated Malassezia on culture. Colony forming unit's per tape (CFU/tape) expressed low density of Malassezia yeasts isolated on Leeming-Notman agar (LNA) from non-lesional skin (NLS) in healthy controls (HC) (A); high density of Malassezia yeasts isolated on Dixon agar from lesional skin (LS) in Parkinson disease (PD) (B) and identification of Malassezia by Tween assimilation test (C).

LS in $53 \%$ of SDP group and in $21.7 \%$ of SDN group (mean CFU/tape $=31.9)(\mathrm{p}<0.01)$. The mean CFU/ tape on NLS was the highest in the SDP (mean CFU/ tape $=44.3)$ followed by SDN (mean CFU/tape $=19.8)$ and $\mathrm{HC}$ (mean CFU/tape $=17.4)(\mathrm{p}<0.01)$. Correlation between studied groups in Malassezia CFU/tape on NLS highlighting significantly higher Malassezia density in SDP group $(\mathrm{p}=0.018)$ (Table 2$)$.

\section{The prevalence of different Malassezia species and their enzyme production}

The prevalence of different Malassezia isolated from NLS and LS in studied groups is shown in Figure 2. The most prevalent species in SDP/LS was M. globosa 11/26 (42.3\%), followed by $M$. furfur $7 / 26$ (26.9\%) and M. obtusa $4 / 26$ (15.4\%). In SDN/LS the most prevalent species was M. slooffiae 14/53 (26. 5\%), followed by M. sympodialis $9 / 53(17 \%)$ and M. globosa 9/53 (17\%) $(\mathrm{p}=0.023)$. The differences were noted only for $M$. globosa (42.3\%; SDP/LS) and M. sloffiae (26.4\%; SDN/LS) ( $<<0.05)$.

On NLS the most common species were: $M$. slooffiae (22\%) in SDN group; M. globosa (25\%) in $\mathrm{HC}$ and $M$. globosa (42.3\%) in PD group. In SDP group the most prevalent species (M. globosa, M. furfur and M. obtusa) were the same on LS and NLS. The observed difference between isolated Malassezia on LS vs. NLS was not statistically significant for SDN and $\mathrm{HC}$ groups (SDN; $\mathrm{p}=0.933),(\mathrm{HC} ; \mathrm{p}=0.541)$ (Figure 2$)$.

The observed data suggest a significant role of infection, presence of different Malassezia species and yeast density in ethiopathogenesis of SD. Therefore, we evaluated the hypothesis that Malassezia yeasts replication and lipases production on the host skin may be important for pathogenesis of SD. Thus we tested (selection of isolates, $\mathrm{n}=26$ ) for production of different enzyme classes in vitro. All phosphatase and lipase enzymes were produced in significant amount from all tested Malassezia isolates. High rate of $\beta$-glucuronidase and leucine arylamidase activity was detected at $M$. furfur isolates only (Table 3).

\section{Correlation between study groups and culture-based mycological findings}

The correlation between studied groups and culture-based mycological findings performed by logistic regression analysis for sex and age showed significant higher intensity of SD in SDN group (OR 0.81; 95\% CI = 0.66-0.99; $\mathrm{p}=0.043$ ) (Table 4). Our data demonstrated predominant male sex in patients with SD and moderate or severe form of SD in both studied groups. There was no difference between studied groups in SD severity regarding categories, but when SD was measured by scale SD severity was a

Table 1 Demographics and description of SD

\begin{tabular}{|c|c|c|c|c|c|}
\hline Variables & Healthy Control $(\mathrm{HC}) \mathrm{n}=70$ & SDN $n=60$ & SDP $n=30$ & Total $n=160$ & $p$ \\
\hline \multicolumn{6}{|l|}{ Demographics } \\
\hline Sex M/F (\%) & $26 / 44(37.1)$ & $38 / 22(63.3)$ & $23 / 7(76.7)$ & $87 / 73(54.4)$ & 0.000 \\
\hline Age & $48.7 \pm 19.9$ & $48.3 \pm 17.9$ & $66.6 \pm 6.3^{*}$ & $51.9 \pm 18.7$ & 0.000 \\
\hline \multicolumn{6}{|l|}{ Description of SD } \\
\hline Intensity & - & $8.65 \pm 6.7$ & $6.9 \pm 2.7$ & $8.1 \pm 5.7$ & 0.175 \\
\hline Intensity in categories & & & & & 0.314 \\
\hline Mild $(\leq 5)$ & - & $15(25)$ & $7(23.3)$ & $22(24.2)$ & \\
\hline Moderate (>5 and < 12) & - & $27(45)$ & $18(60)$ & $45(50)$ & \\
\hline Sever $(\geq 12)$ & - & $18(30)$ & $5(16.7)$ & $23(25.6)$ & \\
\hline
\end{tabular}

Abbreviations: $H C$, Healthy controls; $S D$, Seborrheic dermatitis; $S D N$, Seborrheic dermatitis in patients without Parkinson disease; SDP, Seborrheic dermatitis in patients with Parkinson disease; $M$, Male; $F$, Female; ${ }^{*} p<0.05$. 
Table 2 Comparison of Malassezia colonies number (CFU/tape) on lesional and non-lesional skin between studied groups

\begin{tabular}{|c|c|c|c|c|c|}
\hline \multicolumn{6}{|l|}{ The characteristics of experiment } \\
\hline LS & $\mathrm{HC} n=70$ & SDN $\mathrm{n}=60$ & SDP $\mathbf{n}=30$ & Total $n=160$ & $\mathrm{p}$ \\
\hline Number of colonies (CFU/tape) & - & $31.9 \pm 38.1$ & $67.8 \pm 51.7$ & $43.9 \pm 46.1$ & 0.006 \\
\hline Number of colonies in categories & - & & & & \\
\hline None & - & $7(11.7)$ & $4(13.3)$ & $11(12.2)$ & \\
\hline Low CFU/tape $(\leq 20)$ & & $30(50)$ & $3(10)$ & $33(20.6)$ & \\
\hline Medium CFU/tape (20-49) & - & $10(16.7)$ & $7(23.3)$ & $17(10,6)$ & \\
\hline High CFU/tape ( $\geq 50)$ & - & $13(21.7)$ & $16(53.3)$ & $29(18.1)$ & \\
\hline \multicolumn{6}{|l|}{ NLS } \\
\hline Number of colonies (CFU/tape) & $17.4 \pm 30.1$ & $19.8 \pm 31.1$ & $44.3 \pm 44.7^{* *}$ & $23.4 \pm 34.9$ & 0.018 \\
\hline \multicolumn{6}{|l|}{ Number of colonies in categories } \\
\hline None & $26(37.1)$ & $19(31.7)$ & $4^{* * *}(13.3)$ & $49(30.6)$ & \\
\hline Low CFU/tape $(\leq 20)$ & 27 (38.6) & $23(38.3)$ & $11(36.7)$ & $61(38.1)$ & \\
\hline Medium CFU/tape (20-49) & $9(12.9)$ & $9(15)$ & $4(13.3)$ & $22(13.7)$ & \\
\hline High CFU/tape ( $\geq 50)$ & $8(11.4)$ & $9(15.0)$ & $11(36.7)$ & $28(17.5)$ & \\
\hline
\end{tabular}

Abbreviations: $H C$, Healthy controls; SDN, Seborrheic dermatitis in patients without Parkinson disease; SDP, seborrheic dermatitis in patients with Parkinson disease; $L S$, Lesional skin; NLS, Non-lesional skin; CFU, Colony forming units; ${ }^{* *} \mathrm{p}<0.05 ;{ }^{* * *} \mathrm{p}<0.01$.

significantly higher in SDN group (logistic regression analyses for sex and age) (Table 4). Therefore, a positive correlation between SD, PD, M. globosa, high yeast density and high phosphatase and lipase activity of Malassezia yeasts was observed. This may suggest the possible role of the skin SER in PD patients for yeasts replication and lipases enzyme production.

\section{Discussion}

The concept of skin as a mirror of Parkinsonism dates back to the beginning of the last century. Since then, a good deal of evidence has been accumulated in support of the causal association between the neurological disturbance and changes detectable on areas of the integument with the richest sebaceous gland supply. SD is a common

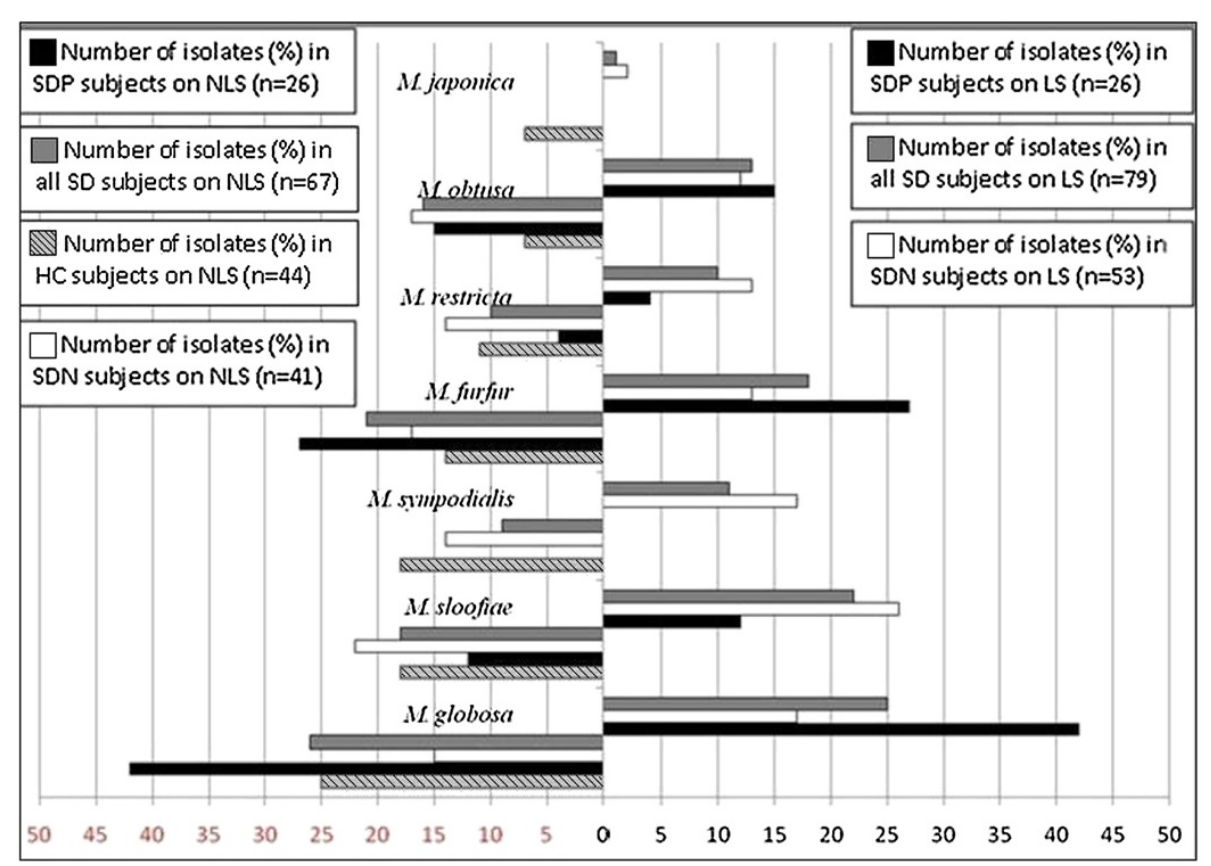

Figure 2 Quantitative graphical presentation of different Malassezia species. Graphical presentation based on the percent of total positive culture on LNA and Dixon media, isolated from non lesional skin (NLS) and lesional skin (LS) on the: (i) all patients with SD; (ii) patients with SD and without Parkinson's disease (SDN); (iii) patients with SD and Parkinson's disease (SDP) and (iv) healthy controls (HC). 
Table 3 Enzymatic activity of different Malassezia species determined by the Api Zym assay

\begin{tabular}{|c|c|c|c|c|c|c|}
\hline Class of enzymes & Type of enzymes & $\begin{array}{l}\text { All strains } \\
\mathrm{n}=26(\%)\end{array}$ & $\begin{array}{l}M . \text { furfur } \\
\mathrm{n}=9(\%)\end{array}$ & $\begin{array}{l}\text { M. globosa } \\
\mathrm{n}=7 \text { (\%) }\end{array}$ & $\begin{array}{l}\text { M. slooffiae } \\
\mathrm{n}=4(\%)\end{array}$ & $\begin{array}{l}\text { M. obtusa } \\
\mathrm{n}=6(\%)\end{array}$ \\
\hline \multirow[t]{5}{*}{ Proteases } & Leucine arylamidase & $11(42)$ & $7(78)$ & $2(29)$ & $1(25)$ & $1(17)$ \\
\hline & Valine arylamidase & $\varnothing$ & $\varnothing$ & $\varnothing$ & $\varnothing$ & $\varnothing$ \\
\hline & Cystine arylamidase & $\varnothing$ & $\varnothing$ & $\varnothing$ & $\varnothing$ & $\varnothing$ \\
\hline & Trypsin & $\varnothing$ & $\varnothing$ & $\varnothing$ & $\varnothing$ & $\varnothing$ \\
\hline & a-Chymotrypsin & $\varnothing$ & $\varnothing$ & $\varnothing$ & $\varnothing$ & $\varnothing$ \\
\hline \multirow[t]{3}{*}{ Lipases } & Lipase (C14) & $14(54)$ & $6(67)$ & $4(57)$ & $3(75)$ & $2(33)$ \\
\hline & Esterase lipase (C8) & $25(96)$ & $9(100)$ & $7(100)$ & $4(100)$ & $5(83)$ \\
\hline & Esterase (C4) & $25(96)$ & $6(67)$ & $4(57)$ & $3(75)$ & $2(33)$ \\
\hline \multirow[t]{3}{*}{ Phosphatases } & Acid phosphatase & $26(100)$ & $7(100)$ & $7(100)$ & $4(100)$ & $6(100)$ \\
\hline & Alkaline phosphatase & $18(69)$ & $7(78)$ & $4(57)$ & $3(75)$ & $3(50)$ \\
\hline & Naphthol-AS-BI- phosphohydrolase & $23(89)$ & $8(89)$ & $7(100)$ & $4(100)$ & $5(83)$ \\
\hline \multirow[t]{8}{*}{ Glycosidase } & $\beta$ - Glucuronidase & $9(35)$ & $3(33)$ & $2(29)$ & $2(50)$ & $2(33)$ \\
\hline & a - Galactosidase & $\varnothing$ & $\varnothing$ & $\varnothing$ & $\varnothing$ & $\varnothing$ \\
\hline & $\beta$ - Galactosidase & $\varnothing$ & $\varnothing$ & $\varnothing$ & $\varnothing$ & $\varnothing$ \\
\hline & N-acetil- $\beta$-glucosaminadase & $\varnothing$ & $\varnothing$ & $\varnothing$ & $\varnothing$ & $\varnothing$ \\
\hline & a - Glucosidase & $\varnothing$ & $\varnothing$ & $\varnothing$ & $\varnothing$ & $\varnothing$ \\
\hline & $\beta$ - Glucosidase & $\varnothing$ & $\varnothing$ & $\varnothing$ & $\varnothing$ & $\varnothing$ \\
\hline & a - Mannosidase & $\varnothing$ & $\varnothing$ & $\varnothing$ & $\varnothing$ & $\varnothing$ \\
\hline & a - Fucosidase & $\varnothing$ & $\varnothing$ & $\varnothing$ & $\varnothing$ & $\varnothing$ \\
\hline
\end{tabular}

Data showed all type of enzymes from lipase and phosphate classes detected in a high percentage from the tested species isolated in this study.

chronic and relapsing inflammatory skin disorder which primarily affects sebum rich areas. The prevalence of SD is $11.6 \%$ in USA [6] but can be higher in elderly people (over 80\%) [5] and in Parkinsonism (52-59.5\%) [8]. In recent years, a few studies concerning SD and PD have been published [18-20]. To our best knowledge, this is the first culture-based epidemiology study performed on patients with SD and PD. In the present study male SDP was dominant and this was in agreement with the study carried out by Gupta who explained the observed result by stronger action of androgens in the male SD patients [20], as well as with the study by Wooten who found a male to female ratio in PD of 1.49 [21]. Mean age of our SDP patients was $67 \pm 7$ years (Table 1) which was in agreement with studies restricted to individuals 65 years or above, confirming that the median incidence rate of PD was considerably higher in group above 60 year old [22].

The severity of SD depends on medical conditions and underlying diseases $[13,11]$. Accordingly, we observed a high

Table 4 Correlation between study groups and culture-based mycological findings expressed as number and presence of Malassezia colonies on lesional and non-lesional skin

\begin{tabular}{|c|c|c|c|}
\hline \multirow{2}{*}{$\begin{array}{l}\text { Variables/potential classificatory } \\
\text { of selected groups }\end{array}$} & \multicolumn{3}{|c|}{ Logistic regression analysis adjusted on age and sex $(\mathrm{OR} ; 95 \% \mathrm{Cl} ; \mathrm{p})$} \\
\hline & SDN vs. SDP & HC vs. SDN & HC vs. SDP \\
\hline Intensity of SD & $0.81 ; 0.66-0.99 ; 0.043^{*}$ & - & - \\
\hline Intensity of SD in categories & $0.46 ; 0.20-1.04 ; 0.063$ & - & - \\
\hline Number of colonies (CFU/tape) on LS & $1.01 ; 0.99-1.02 ; 0.110$ & - & - \\
\hline Number of colonies (CFU/tape) on LS by categories & $1.51 ; 0.62-3.64 ; 0.363$ & - & - \\
\hline Number of colonies (CFU/tape) on NLS & $1.01 ; 0.99-1.02 ; 0.398$ & $0.99 ; 0.98-1.01 ; 0.786$ & $1.01 ; 0.99-1.02 ; 0.288$ \\
\hline Number of colonies (CFU/tape) on NLS by categories & $1.35 ; 0.56-3.29 ; 0.504$ & $0.98 ; 0.54-1.78 ; 0.954$ & $1.60 ; 0.75-3.42 ; 0.225$ \\
\hline Presence of Malassezia on LS & $0.89 ; 0.17-4.68 ; 0.898$ & - & - \\
\hline Presence of Malassezia on NLS & $1.89 ; 0.45-8.02 ; 0.387$ & $0.97 ; 0.44-2.12 ; 0.933$ & $2.32 ; 0.63-8.53 ; 0.206$ \\
\hline
\end{tabular}

Abbreviations: $O R$, Odds ratio; $C l$, Confidence interval; $S D$, Seborrheic dermatitis; $S D N$, Seborrheic dermatitis in patients without Parkinson disease; $S D P$, Seborrheic dermatitis in patients with Parkinson disease; HC, Healthy controls; LS, Lesional skin; NLS, Non-lesional skin; CFU, Colony forming units; ${ }^{*} \mathrm{p}<0.05$. 
number of SDP with moderate or severe SD form and a high number of positive cultures (Table 2). Our data could be explained by predominant male gender and an increased level of SER and MSH secretion in PD patients [23].

Our culture-based study demonstrated six different Malassezia isolated in SDP patients, with M. globosa $(42.3 \%)$ as the most dominant species (Figure 1). Several studies identified M. globosa as the most prevalent species in SD, suggesting its principal role in SD [24] but data for patients with PD have not been reported yet. By comparing the severity of SD and Malassezia presence on LS we did not find a significant difference between studied groups. By contrast, using the laboratory based quantitative test for Malassezia yeasts density (expressed as CFU/tape) we found more than twice higher CFU/tape for SDP/LS than SDN/LS group. Literature data are controversial; some studies showed that the density increases with the intensity of skin lesions, while other studies were unable to confirm this result [10]. However, the reduction of Malassezia yeast density always results in SD outcome improvement [14].

The effects of the anti-Parkinson's agent L-dopa on SD have been reportedly evaluated in Parkinson's and data showed that treatment with L-dopa restores MHCinhibiting factor synthesis and reduces sebum secretion in Parkinson's [25]. Our patients were treated with L-dopa during the course of this study so this can be reason for the similarity in the clinical findings between different groups, even high SD intensity in SDN group (Table 4). However, other factors contribute to SD development such as facial immobility ("mask-like face") which may lead to an increased sebum accumulation.

The pathogenesis of SD is still not completely understood but some clinical studies indicated that Malassezia presence and replication plays a pivotal role. Positive clinical response of SD to antifungals (i.e., azoles, ciclopirox) could be explained by their role in reduction of Malassezia yeast proliferation, again confirming an important role of Malassezia density and infection in pathogenesis of SD.

Malassezia requires exogenous specific lipids for growth, therefore tends to appear on skin around the time of puberty, when there is an increase in androgens and consequently an elevation in sebum production [12]. In patients with SD triglycerides and cholesterol are usually elevated but squalene and free fatty acids were significantly decreased compared to normal controls. Free fatty acids are formed from triglycerides through the action of bacterial lipases such as Propionibacterium acnes. This suggests that an imbalance of microbial flora and alterations in the composition of skin surface lipids may be involved [26].

Malassezia represents a part of human flora normally colonizing the skin surface but in some conditions it can change its saprophytic state and invade stratum corneum. Some culture-based laboratory studies identified differences between NLS and LS in the percentage of negative culture or in the presence and density of Malassezia [13]. We did not find significant difference in the absence of Malassezia on NLS between HC and SDN, but very low number of negative Malassezia cultures was detected on NLS in SDP. It may suggest an important role of the host environment such as the SER in PD group which may provide favorable conditions for expressing higher virulence capacity of Malassezia. Recent study showed a possible role of lipase in the host environment to produce free fatty acids, which are important for enhancing the Malassezia virulence [27]. The mechanisms of the Malassezia transition from commensal to pathogen are not clear yet, but this evidence is important for further studies due to the fact that lipases are further involved in the release of arachidonic acid, which can be important in cutaneous inflammation and disease [28].

It is well known that the development of infections depends on microbe's replication while the severity of disease may depend of microbe's enzyme production. In pathogenicity of SD phosphatase and lipase yeasts enzymes can initiate inflammatory response by releasing oleic and arahidonic acids from the sebum lipids [12,28]. Fatty acids have direct irritant and desquamative effects on keratinocytes, while arahidonic acid metabolized by cyclooxigenase serves as a source of pro-inflammatory eicosanoides and leads to inflammation and damage to stratum corneum. Our strains tested for different enzymes classes' expressed all type of phosphatase and lipase enzymes manly at a high level (Table 4). These findings suggest that the infection, yeasts replication and density are key pathogenic mechanisms in SD and can contribute to the development of Malassezia-driven pathogenic "vicious circle", which closes due to the fact that saturated fatty acids, released by Malassezia lipase, are used as a "proliferative fuel" for these yeasts. M. globosa showed the highest lipase activity, suggesting that lipase could be a pathogenic factor in the skin diseases associated with different Malassezia and providing an explanation about M. globosa as the most important pathogenic species in PD patients with SD [29].

Literature data suggest the role of another mechanism in pathogenicity of SD. Beside infection the allergy host reaction to Malassezia antigens can be involved in SD. In atopic patients, some enzymes from protease and glycosidase classes, such as $\beta$-glucuronidase and leucine arylamidase, may act as antigens. Thus, genetic predisposition, as well as Malassezia antigens can lead to the local skin immune response and immunopathology and to development of SD $[12,29,30]$. In our study we clearly demonstrated very low impact of enzymes which may act as Malassezia antigens (Table 3). 


\section{Conclusions}

This is the first culture-based epidemiology study performed on patients with SD and PD. The data demonstrated a positive correlation between SD, PD and Malassezia presence and density. Our findings that SDP group had high yeast density and associated high phosphatase and lipase activity in vitro may lead to conclusion that infection is the key mechanism of SD in PD patients. Future better understanding of the interactions between different Malassezia and PD host may provide new opportunities for improved SD control in PD. The appropriate antifungal treatment can be useful for PD patients. Antifungals may reduce Malassezia growth and their enzymes production overall improving patients well-being and quality of life. Correlation between reduction of Malassezia density, positive outcome of SD and the reduction of the non-motile symptoms in PD patients need to be further investigated.

\section{Abbreviations}

SD: Seborrheic dermatitis; PD: Parkinson's disease; SDP: Patients with SD and PD; SDN: Patients with SD without PD; HC: Healthy controls; LS: Lesional skin; NLS: Non-lesional skin; CFU/tape: Colony forming units per tape; SER: Sebum excretion ratio; MSH: Melanocyte-stimulating hormone.

\section{Competing interests}

The authors declare that they have no competing interests.

\section{Authors' contributions}

VAA and DM were involved in study design, data collection, and analysis and results interpretation. $\mathrm{DM}$ and $\mathrm{AB}$ had done laboratory tests and data presentation. BV and JM were involved in data correlation and interpretation. VAA and VK were responsible for laboratory and clinical part of the study, had advisory and supervisory role and were responsible for important intellectual content. All authors drafted the manuscript and made substantial contributions to the revised manuscript. All authors read and approved the final manuscript.

\section{Acknowledgment}

The paper has been published with the supports of the Projects of Ministry of Education, Science and Technology of the Republic of Serbia (grants No Ol 175034 and No Ol 175033) and Pfizer H.C.P. Corporation, Serbia. We thank Dr Milena Radulović (University of Belgrade School of Dental Medicine, Belgrade, Serbia), Dr Jasmina Nikodinović Runić (Laboratory of Molecular Genetics and Microbial Ecology, University of Belgrade Institute of Molecular Genetics and Genetic Engineering) and Smiljka Surla (London, UK) for correction of English language in the manuscript.

\section{Author details}

IInstitute of Microbiology and Immunology, University of Belgrade Medical School, Dr Subotica 1, Belgrade, Serbia. ${ }^{2}$ Department of Dermatology, Dermatology Unit, Clinical Center of Serbia; Military Medical Centre, Belgrade, Serbia. ${ }^{3}$ Management School, Alfa University, Belgrade, Serbia. ${ }^{4}$ Institutes for Statistics and Medical Informatics, University of Belgrade School of Medicine, Belgrade, Serbia. Institute of Neurology Clinical Centre of Serbia, University of Belgrade, School of Medicine, Belgrade, Serbia.

Received: 2 September 2013 Accepted: 28 February 2014 Published: 14 March 2014

\section{References}

1. Yao SC, Hart AD, Terzella MJ: An evidence-based osteopathic approach to Parkinson disease. Osteopath Fam Physician 2013, 5:96-101.
2. de Lau LM, Breteler MM: Epidemiology of Parkinson's disease. Lancet Neurol 2006, 5:525-535.

3. Kostić VS: Treatment of young-onset Parkinson's disease: role of dopamine receptor agonists. Parkinsonism Relat Disord 2009, 15:71-75.

4. Shuster S, Thody AJ, Goolamali SK, Burton JL, Plummer N, Bates D: Melanocyte-stimulating hormone and parkinsonism. Lancet 1973, 3:463-464.

5. Binder RL, Jonelis FJ: Seborrheic dermatitis in neuroleptic induced Parkinsonism. Arch Dermatol 1983, 119:473-475.

6. Naldi L, Rebora A: Clinical practice: seborrheic dermatitis. N Engl J Med 2009, 360:387-396.

7. Marino CT, McDonald E, Romano JF: Seborrheic dermatitis in acquired immunodeficiency syndrome. Cutis 1991, 50:217-218.

8. Braak H, del Tredici K: Non-dopaminergic pathology in Parkinson's disease. In The Non-Motor and Non-Dopaminergic Features of Parkinson's Disease. Edited by Olanow CW, Stocchi F, Lang AE. Oxford: Blackwell Publishing Ltd; 2011:40-41.

9. Mastrolonardo M: Seborrheic dermatitis, increased sebum excretion and Parkinson's disease: a survey of (im)possible links. Med Hypotheses 2003, 60:907-911.

10. Ashbee HR, Evans EGV: Immunology of disease associated with Malassezia species. Clin Microbiol Rev 2002, 15:21-57.

11. Gaitanis G, Magiatis P, Hantschke M, Bassukas ID, Velegraki A: The Malassezia genus in skin and systemic diseases. Clin Microbiol Rev 2012, 25:106-141.

12. Gupta AK, Batra R, Bluhm R, Boekhout T, Dawson TL Jr: Skin diseases associated with Malassezia species. J Am Acad Dermatol 2004, 51:785-798.

13. Gupta AK, Kohli Y, Summerbell RC, Faergemann J: Quantitative culture of Malassezia species from different body sites of individuals with or without dermatoses. Med Mycol 2001, 39:243-251.

14. Pechère M, Krischer J, Remondat C, Bertrand C, Trellu L, Saurat JH: Malassezia spp. carriage in patients with seborrheic dermatitis. J Dermatol 1999, 26:558-561.

15. Koca R, Altinyazar HC, Esturk E: Is topical metronidazole effective in seborrheic dermatitis? A double blind study. Int I Dermatol 2003, 42:632-635.

16. Pechère $M$, Remondat $C$, Bertrand $C$, Didierjean $L$, Saurat JH: A simple quantitative culture of Malassezia spp. in HIV-positive persons. Dermatology 1995, 191:348-349.

17. Guillot J, Guého E, Lesourd M, Midgley G, Chevrier G, Dupont B: Identification of Malassezia species. J Mycol Med 1996, 6:103-110.

18. Aubin F, Blanc D, Guinchard C, Agache P: Absence of minocycline in sebum? J Dermatol 1989, 16:369-373.

19. Villares JC, Carlini EA: Sebum secretion in idiopathic Parkinson's disease: effect of anticholinergic and dopaminergic drugs. Acta Neurol Scand 1989, 80:57-63.

20. Gupta AK, Kohli Y: Prevalence of Malassezia species on various body sites in clinically healthy subjects representing different age groups. Med Mycol 2004, 42:35-42.

21. Wooten GF, Currie LJ, Bovbjerg VE, Lee JK, Patrie J: Are men at greater risk for Parkinson's disease than women? J Neurol Neurosurg Psychiatry 2004, 75:637-639.

22. Hirtz D, Thurman DJ, Gwinn-Hardy K, Mohamed M, Chaudhuri AR, Zalutsky R: How common are the "common" neurologic disorders? Neurology 2007, 68:326-337.

23. Martignoni E, Godi L, Pacchetti C, Berardesca E, Vignoli GP, Albani G, Mancini F, Nappi G: Is seborrhea a sign of autonomic impairment in Parkinson's disease? J Neural Transm 1997, 104:1295-1304.

24. Rincon S, Celis A, Sopo L, Motta A, Cepero de Garcia MC: Malassezia yeast species isolated from patients with dermatologic lesions. Biomedica 2005, 25:189-195.

25. Milani M, di Molfetta SA, Gramazio R, Fiorella C, Frisario C, Fuzio E, Marzocca V, Zurilli M, di Turi G, Felice G: Efficacy of betamethasone valerate $0.1 \%$ thermophobic foam in seborrhoeic dermatitis of the scalp: an openlabel, multicentre, prospective trial on 180 patients. Curr Med Res Opin 2003, 19:342-345.

26. Bolognia JL: Dermatology. In Dermatology: 3e Expert Consult Premium Edition Edited by Bolognia JL, Jorizzo JL, Schaffer JV. Spain: Elsevier; 2012:75-77.

27. Lee YW, Lee SY, Jung WH: Evaluation of expression of Lipases and Phospholipases of Malassezia in Patient with Seborrheic Dermatitis. Ann Dermatol 2013, 25:310-314.

28. Juntachai W, Oura T, Kajiwara S: Purification and characterization of a secretory lipolytic enzyme, MgLIP2, from Malassezia globosa. Microbiology 2011, 157:3492-3499. 
29. Juntachai W, Oura T, Murayama SY, Kajiwara S: The lipolytic enzymes activities of Malassezia species. Med Mycology 2009, 47:477-484.

30. de Angelis YM, Gemmer CM, Kaczvinsky JR, Kenneally DC, Schwartz JR, Dawson TL Jr: Three etiologic facets of dandruff and seborrheic dermatitis: Malassezia fungi, sebaceous lipids and individual sensitivity. J Investig Dermatol Symp Proc 2005, 10:295-297.

doi:10.1186/1471-5945-14-5

Cite this article as: Arsic Arsenijevic et al:: A laboratory-based study on patients with Parkinson's disease and seborrheic dermatitis: the presence and density of Malassezia yeasts, their different species and enzymes production. BMC Dermatology 2014 14:5.

\section{Submit your next manuscript to BioMed Central and take full advantage of:}

- Convenient online submission

- Thorough peer review

- No space constraints or color figure charges

- Immediate publication on acceptance

- Inclusion in PubMed, CAS, Scopus and Google Scholar

- Research which is freely available for redistribution 